\title{
Cipango
}

Cipango Cahiers d'études japonaises

\section{La Commission d'enquête sur la pollution minière d'Ashio de 1897 et ses enjeux : du laissez-faire à la prise en main étatique d'une crise environnementale}

The Ashio Pollution Investigation Commission of 1897: from Laissez-Faire to the Governmental Control of an Environmental Crisis

\section{Cyrian Pitteloud}

\section{(2) OpenEdition}

\section{Journals}

\section{Édition électronique}

URL : https://journals.openedition.org/cipango/3833

DOI : $10.4000 /$ cipango.3833

ISSN : 2260-7706

\section{Éditeur}

INALCO

Édition imprimée

Date de publication : 1 juillet 2020

ISBN : 9782858313426

ISSN : $1164-5857$

\section{Référence électronique}

Cyrian Pitteloud, «La Commission d'enquête sur la pollution minière d'Ashio de 1897 et ses enjeux : du laissez-faire à la prise en main étatique d'une crise environnementale », Cipango [En ligne], 23 | 2020, mis en ligne le 16 juin 2020, consulté le 30 juin 2021. URL : http://journals.openedition.org/cipango/ 3833 ; DOI : https://doi.org/10.4000/cipango.3833 


\title{
La Commission d'enquête sur la pollution minière d'Ashio de 1897 et ses enjeux : du laissez-faire à la prise en main étatique d'une crise environnementale
}

\author{
The Ashio Pollution Investigation Commission \\ of 1897: from Laissez-Faire to the Governmental \\ Control of an Environmental Crisis
}

Cyrian Pitteloud

Université de Genève

La ville minière d'Ashio, dans les montagnes du département de Tochigi, a donné son nom à l'un des premiers cas de pollution industrielle du Japon ${ }^{1}$. À la fin

1. L'« affaire de pollution de la mine de cuivre d'Ashio » (Ashio dōzan kōdoku jiken 足尾銅山鉱毒事件), ou 《 affaire de pollution minière d'Ashio »(Ashio kōdoku jiken 足尾鉱毒事件), que nous choisissons de raccourcir par 《 affaire d'Ashio », a été abondamment étudiée dès les années 1970. Pour les ouvrages japonais, citons principalement Kano, 1974 ; SHōjI \& SUGAI, 2014 [1984]. Certains articles en anglais, bien que datés, fournissent une synthèse toujours utile, notamment Notehelfer, 1975. On dispose enfin de riches compilations de sources, parmi lesquelles UCHIMIzU, 1971 ; TOCHIGI-KENSHI HENSAN IINKAI, 1980. 
du XIX ${ }^{\mathrm{e}}$ siècle, la modernisation technologique et l'exploitation intensive de son gisement de cuivre ${ }^{2}$ provoquèrent de graves dégâts sur l'environnement du nordest du pays : déforestation, fumées toxiques, empoisonnement des cours d'eau et, à travers ceux-ci, contamination des terres agricoles. Au plus fort de la crise, jusqu'à cinq départements furent touchés. À partir de 1890, la population de la région organisa un mouvement de protestation, qui atteignit son pic au tournant du siècle et se poursuivit jusqu'à la veille des années 1910, avec une intensité variable ${ }^{3}$. La durée de cette lutte, son caractère pluriel, la dimension stratégique du gisement d'Ashio - devenu l'un des premiers fournisseurs de cuivre du pays - et la gravité des dégât,s dont les conséquences persistent encore à l'heure actuelle, en font une des crises écologiques et sociales majeures du Japon moderne.

À rebours d'une historiographie en grande partie axée sur le mouvement de contestation, nous prenons le parti de nous intéresser à l'un des autres acteurs fondamentaux du conflit, à savoir le gouvernement. Le rôle de ce dernier, en effet, a peu été étudié, notamment en ce qui concerne les années 1896 et $1897^{4}$, durant lesquelles le pouvoir central décida de s'impliquer davantage après une décennie de laissez-faire. Nous essayerons de comprendre de quelle façon celui-ci négocia la poursuite des activités minières, malgré le degré élevé de conflictualité qui présida à ce moment où le désastre atteignait des proportions qu'il n'était plus possible d'ignorer. À cet égard, la mise sur pied en mars 1897 d'une Commission d'enquête sur la pollution minière d'Ashio (tableau 1) représente une étape décisive.

Si les travaux de cette Commission de 1897 sont effectivement retenus par les historiens comme le début de l'action gouvernementale - voire parfois, dans une perspective plus large, comme une avancée notable dans l'histoire de la protection de l'environnement -, ils n'ont paradoxalement suscité que de rares études, et

2. Sur la mine, voir entre autres MURAKAMI, 2006. Quant aux aspects propres aux conflits du travail à Ashio : Nimura, 1988. L'ouvrage a été partiellement traduit en anglais : Nimura, 1997.

3. Partie d'une protestation locale, la mobilisation s'étendra à la métropole et impliquera des milieux socioculturels fort variés.

4. Ce constat a amené Konishi Tokuō à produire, entre 1989 et 1990, trois articles fondamentaux sur cette période : Konishi, 1989, 1990a \& 1990b. 
sont généralement traités en termes peu nuancés ${ }^{5}$. Les dispositions adoptées par le gouvernement à la suite des délibérations de la Commission ne réglèrent pas le problème : elles ne furent essentiellement qu'un moyen de garantir la poursuite des activités minières tout en donnant le change au mouvement de protestation ${ }^{6}$, même si elles sont encore souvent présentées comme une prise de mesures fermes à l'encontre de l'exploitant ${ }^{7}$. Dès lors, il nous paraît essentiel de revenir sur ce moment charnière, qui s'étend de la fin de l'année 1896 au milieu de l'année 1897, et plus particulièrement sur les discussions entre les commissaires. Tout en étant un organe interministériel, temporaire et limité à un rôle consultatif, la Commission apparaît, à la lecture de ses procès-verbaux ${ }^{8}$, traversée par des tensions et des affrontements qui révèlent des enjeux bien plus larges ; ceux-là même auxquels fut confronté le tout nouvel État centralisé, alors en train de mettre en place sa structure administrative et d'industrialiser le pays à marche forcée aux dépens de l'environnement, et par conséquent, de l'agriculture. Si des nuisances existaient déjà avant l'ère Meiji ${ }^{9}$, la pollution prit cependant une ampleur encore non égalée avec le développement de l'industrie, en même temps qu'elle devint plus médiatisée, notamment par le biais de l'affaire qui nous intéresse ici.

5. La recherche n'a été que peu renouvelée depuis les travaux de Konishi, bien que d'autres éditions de sources aient été publiées autour des années 2000 : KōDOKUSHI HENSAN IINKAI, 2006-2013; ANZAI et al., 2009.

6. Ce point de vue, auquel nous adhérons, est défendu par des chercheurs comme Shōji et Sugai, mais ce sont surtout les articles de Konishi qui le démontrent de façon convaincante.

7. Dernier exemple en date : Miura, 2017. L'auteur de cet ouvrage, pourtant soigneusement documenté, évoque brièvement les divergences des commissaires, mais s'attarde principalement sur l'arrêté émis à la suite des délibérations de la Commission, essentiellement pour en souligner la sévérité.

8. Les minutes sont publiées dans « Ashio dōzan kōdoku jiken chōsa iinkai sokkiroku $($ shō $) 》$ 足尾銅山鉱毒事件調査委員会速記録（抄）[Procès-verbaux de la commission d'enquête sur la pollution minière d'Ashio. Extraits], ci-après « Ashio chōsa sokkiroku », in Tochigi-Kenshi hensan IInkAI, 1980, p. 641-813. Bien que légèrement abrégée par endroits, cette version offre l'avantage d'une retranscription. Les minutes complètes, manuscrites, sont librement consultables via le site des Archives nationales du Japon : https://www.digital.archives.go.jp/.

9. ANDŌ, 1992. 


\section{CIPANGO \\ 210 Cahiers d'études japonaises $n^{\circ} 23$}

Les incidents de ce genre ne se limitèrent pas au seul cas d'Ashio ${ }^{10}$, et analyser l'action du gouvernement dans ce cas précis révèle à la fois comment la pollution industrielle fut abordée à l'époque moderne, et de quelle manière une expertise se forma sur les questions environnementales.

\section{6 - le conflit s'aggrave et l'État est contraint d'intervenir}

En 1896, une suite d'événements force le gouvernement à s'impliquer davantage. Le 21 juillet, le 17 août et le 8 septembre, de graves inondations frappent les départements de Tochigi et de Gunma. La contamination des eaux amplifie les dégâts et se propage aux départements de Saitama, Ibaraki, Chiba, ainsi qu'au bassin du fleuve Edo, sur le territoire de Tōkyō. La population, qui s'était déjà soulevée en 1890, se mobilise à nouveau. Devant l'inaction des autorités locales, celle-ci organise des « marches» (oshidashi 押出し) à destination de la capitale afin de remettre directement ses requêtes aux autorités centrales.

Contrairement aux années précédentes, le gouvernement ne peut cette fois rester inactif. Le 11 novembre 1896, le deuxième cabinet de Matsukata Masayoshi 松方正義 (1835-1924) détache l'ingénieur agronome Sakano Hatsujirō 坂野初 次郎 (1867-1903) ${ }^{11}$ pour des inspections dans les zones contaminées de Gunma et de Tochigi, ainsi qu'à la mine. Le gouvernement demande de surcroît aux autorités des deux départements précités des rapports d'enquête. Au vu des informations récoltées, une première « Commission spéciale d'enquête sur la pollution minière d'Ashio »(Ashio dōzan ködoku tokubetsu chōsa iin-kai 足尾銅山鉱毒特別調 査委員会) est instaurée le 22 décembre au sein du ministère de l'Agriculture et

10. Voir par exemple Shimizu, 1995. Peu d'écrits sont disponibles en anglais sur les autres cas de pollution. Pour une introduction, voir Morris-SuzUKI, 1998.

11. Diplômé de la section de chimie agricole de la Faculté d'agronomie de l'université impériale (Tōkyō) en 1890. D’abord chargé par le ministère de l’Agriculture et du Commerce d'améliorer la technologie de l'industrie sucrière du département de Kagoshima, en 1891 et 1892, il est détaché au laboratoire expérimental d'agriculture du même ministère pour réaliser des analyses dans le cadre de l'affaire d'Ashio. Pour les membres de la Commission, voir le tableau 1. 
du Commerce ${ }^{12}$. Le 25 décembre, elle remet au ministre Enomoto Takeaki 榎 本武明 (1836-1908) un rapport (tōshinsho 答申書) faisant état du déversement de 《substances nocives 》 (yügai busshitsu 有害物質) dans la rivière Watarase, et de dégâts portés aux terres agricoles. Jusqu'alors, les mesures adoptées par l'exploitant de la mine Furukawa Ichibē 古河市兵衛 (1832-1903) ${ }^{13}$ s'étaient limitées à l'installation, en 1893 , d'extracteurs de poussières de minerai ${ }^{14}$ et de bassins de précipitation. Reconnaissant l'insuffisance de ces précautions, le rapport préconise d'améliorer le traitement des eaux usées de la mine et le stockage des déblais, du sable et des stériles, pour éviter qu'ils ne se déversent dans les cours d'eau.

12. Murakami, 2006, p. 201 ; Shōji \& Sugai, 2014 [1984], p. 56.

13. Furukawa eut d'abord un certain succès dans le négoce de soie grège au sein du groupe Ono (Onogumi 小野組). En 1871, il maria sa fille au second fils de Mutsu Munemitsu 陸奥宗光 (1844-1897) et en fit son héritier légal. Mutsu fut notamment gouverneur de Hyōgo, puis de Kanagawa ou encore ministre de l'Agriculture et du Commerce en 1890. Bénéficiant de nombreux appuis, Furukawa fit rapidement fortune dans le secteur minier. Il acheta plusieurs gisements, comme ceux d'Innai 院内 et d'Ani 阿仁, qu'il développa et exploita adroitement. À la fin du siècle, il dirigeait un véritable empire, essentiellement basé dans l'Est et le Nord-Est du pays. Il possédait en 1897 douze mines de cuivre (fournissant environ $40 \%$ de la production domestique), huit mines d'argent, une mine d'or, ainsi que de nombreuses autres entreprises. Dans les décennies suivantes, les activités du groupe, qui devint un véritable conglomérat (zaibatsu 財閥), s’étendirent à l'industrie lourde, la chimie et la machinerie.

14. Dans le cadre d'《 arrangements à l'amiable 》(jidan keiyaku 示談契約) qu'il conclut avec une partie des habitants dès 1892, Furukawa présenta ces équipements comme un moyen de lutter contre la pollution. En échange de modiques sommes d'argent et de sa promesse de réduire les nuisances grâce à ces extracteurs, les signataires s'engageaient à ne plus se plaindre pendant une période d'essai de quatre ans. En réalité, l'appellation d'《extracteurs de poussière »(funtō saishūki 粉鉱採集器) regroupait diverses installations de tri et d'élimination de la boue, des résidus et du sable, dans le but principal d'accroître la production. Selon l'historien Nimura Kazuo, des documents de 1897 montrent clairement l'efficacité limitée de ces appareils en termes de taux de récupération. Cette mesure avait bien peu à voir avec une quelconque diminution des rejets toxiques. Nimura, 1988, p. 214-215. 
212 Cahiers d'études japonaises $n^{\circ} 23$

On ne considère pas qu'une interruption des opérations de la mine soit pour autant nécessaire ${ }^{15}$. Officiellement, celle-ci ne changerait rien à la contamination, notamment aux dépôts au fond du fleuve. La solution préconisée est essentiellement technique, et à deux reprises, il est affirmé que des travaux préventifs permettront d'éviter de nouveaux dégâts. La Commission spéciale suggère plusieurs contremesures telles la désinfection à la chaux, ou le retranchement de la terre contaminée, pour tenter de sauver les terrains cultivables. Elle prescrit en outre le reboisement ainsi qu'une meilleure gestion fluviale, reconnaissant ainsi un lien entre la déforestation pratiquée pour alimenter la mine et les inondations. C'est donc un exposé très complet, qui a non seulement le mérite de soulever les principales difficultés relatives à la pollution, mais qui aborde en outre la question des réparations financières : en plus de couvrir les frais de construction des ouvrages de prévention, l'exploitant devrait en effet indemniser les victimes. La Commission propose enfin de charger les autorités locales de servir d'intermédiaire entre la population et Furukawa, notamment pour établir le montant des dédommagements. Son rapport prouve qu'en 1896, les autorités n'ignoraient pas l'étendue et les détails du problème.

Pourquoi le gouvernement est-il réticent à intervenir ? Il faut d'abord rappeler l'importance du secteur minier qui, après la Révolution de Meiji, fait l'objet d'une attention particulière, alors qu'une grande partie des mines devient propriété étatique. Entre 1870 et 1885, il s'agit en effet du deuxième poste de dépenses ordinaires du ministère de l'Industrie après les chemins de fer (respectivement 31,5\% et $49,9 \%)^{16}$. Le gouvernement ne se contente pas de développer le secteur minier : il encourage la formation dans ce domaine à travers des écoles professionnelles, importe des machines, engage des ingénieurs étrangers, et promeut plus généralement le renouvellement technologique. Durant les décennies suivantes, la production va considérablement augmenter : entre 1874 et 1908, elle est multipliée par 20 pour le cuivre, par 45 pour l'argent, par 54 pour l'or et par 72 pour le charbon ${ }^{17}$. À partir des

15. 《Ashio dōzan kōdoku tokubetsu chōsa iin-kai tōshin-sho »足尾銅山鉱毒特別 調査委員会答申書 (Rapport de la Commission spéciale d'enquête sur la pollution minière d'Ashio), in Tochigi-Kenshi HensAn IINKAI, 1980, p. 629.

16. SiPPEL, 2006, p. 16.

17. Ibid., p. 10. 
années 1880 , le gouvernement procède à une large privatisation des sites, notamment dans le but de réduire ses frais de fonctionnement. À titre d'exemple, entre 1882 et 1886, les mines d'État fournissent 47,2\% de la production d'or, 30,9\% de celle d'argent, $21,1 \%$ de celle de charbon et seulement $2,9 \%$ de celle de cuivre ${ }^{18}$. Les principales mines de cuivre sont donc dirigées par des acteurs privés.

L'entreprise de Furukawa s'affirme rapidement comme un poids lourd parmi les grands groupes qui bâtissent leur richesse sur des ressources minérales fortement demandées. La mine d'Ashio, dont elle acquiert les droits d'exploitation, s'illustre par sa productivité, s'imposant comme l'un des piliers de l'approvisionnement en cuivre du Japon. Ashio, exploitée officiellement depuis le XVII ${ }^{\mathrm{e}}$ siècle, produisait en 1876 environ trente tonnes de cuivre annuelles pour un déficit estimé à 6000 yens $^{19}$. Une année après le changement d'opérateur, la production se monte déjà à quarante-six tonnes, mais la mine fonctionne toujours à perte. Une pleine rentabilité sera atteinte seulement en 1883. Cette année-là, la quantité de minerai dépasse légèrement les 645 tonnes. La découverte de riches filons entre 1882 et 1884 marque une nouvelle étape, et en 1885, la production avoisine les 4090 tonnes, soit près de 90 fois la quantité de 1877. Elle passera à 7500 tonnes en 1891. Par la suite, les quantités se maintiendront entre cinq et six mille tonnes annuelles ${ }^{20}$. Ainsi, dès 1884, et jusque vers 1904, Furukawa fournit à lui seul entre 35 à $40 \%$ de la production nationale de cuivre, dont l'essentiel provient d'Ashio (entre $75 \%$ et $85 \%$ pour cette même période) ${ }^{21}$. Si le cuivre est indispensable à l'électrification du pays (câbles, fils télégraphiques) ainsi qu'à la fabrication de munitions ${ }^{22}$, il est principalement exporté : entre 1877 et 1897, la part dédiée à l'exportation est d'environ $67,5 \%$ et ne descend jamais au-dessous de $30 \%$, à la fois afin d'équilibrer la balance commerciale, mais aussi

18. TAKAGI, 1974, p. 10.

19. ITSUKA-KAI, 1926, p. 99.

20. Ces chiffres sont tirés de Sōgyō HyAKunENSHI HENSAN IINKAI, 1976, p. 82.

21. Shōji \& Sugai, 2014 [1984], p. 1315.

22. Il faut rappeler que l'affaire d'Ashio se déroule entre deux conflits d'importance pour le pays : la première guerre sino-japonaise de 1894-1895 et la guerre russo-japonaise de 1904-1905. 
d'obtenir des devises étrangères qui serviront à leur tour à l'achat de technologies occidentales ${ }^{23}$. En tant que troisième produit-phare d'exportation dont il représente $5 \%$ de la valeur totale, après la soie grège et le thé ${ }^{24}$, le cuivre acquiert une valeur stratégique dans le processus de développement de l'État moderne et du capitalisme japonais.

Furukawa, qui n'avait jamais été inquiété directement, reçoit toutefois en décembre 1896 un « arrêté » (meirei 命令) du ministère de l'Agriculture et du Commerce, qui lui enjoint la construction d'ouvrages de prévention sans pour autant fixer de délai à leur réalisation. Quels seront les effets de cette décision, finalement très modérée au regard des nombreux aspects problématiques relevés dans le rapport de la Commission spéciale ? À en croire les mémoires de Furukawa, l'industrie minière se serait immédiatement attelée à la tâche ${ }^{25}$, réalisant rapidement des bassins de sédimentation supplémentaires et des espaces de stockage des terrils. Ces mémoires, néanmoins, ne précisent pas à quel moment les ouvrages furent achevés. La véritable efficacité de ce premier train de mesures demeure inconnue, et l'on ignore de surcroît si les autorités procédèrent à un suivi, ce qui ne semble pas avoir été le cas. Plusieurs mois après, les investigations de la deuxième Commission attesteront de nombreuses négligences de la part de la mine. Ce premier arrêté du ministère ne donna donc que des résultats extrêmement limités.

Quelques semaines plus tard, la Diète se penche à nouveau sur la question. Le 26 février, le député de Tochigi Tanaka Shōzō 田中正造 (1841-1913) ${ }^{26}$ présente une interpellation : les extracteurs de poussière sont inefficaces et la pollution

23. Estimation basée sur les chiffres fournis par TAKAGI, 1974, p. 7.

24. Entre 1886 et 1895 . Pour la même période, le charbon représente $6 \%$ des exportations. SiPpel, 2006, p. 10-12.

25. ITSUKA-KAI, 1926, p. 232.

26. Notable de la région, il avait été élu dès la première Diète (1890), et sera réélu à six reprises successives jusqu'en 1901. L'essentiel de son activité parlementaire se concentre sur le sort de la population affectée par la pollution. Les ouvrages consacrés à l'affaire d'Ashio, dont il fut une figure majeure, lui réservent en général une place de choix. Voir ainsi Komatsu, 2001. En français, on lira Souyri, 2016, p. 343-363. 
touche à présent cinq départements, ainsi qu'une partie du territoire de Tōkyōo ${ }^{27}$. Que compte faire le gouvernement ? Tanaka, dans l'un de ces réquisitoires dont il est coutumier, critique les autorités locales, qu'il accuse de favoriser Furukawa, et demande des mesures, en appelant au droit des citoyens à la « protection de la vie et des biens » (seimei zaisan kenri 生命財産権利). Une nouvelle fois, Tanaka invoque la défense de la propriété privée, garantie par la Constitution, pour réclamer une aide aux citoyens spoliés de leurs revenus agricoles. Il conclut en se référant à l'article 19 de la « Loi sur les activités minières » (kōgyō jōrei 鉱

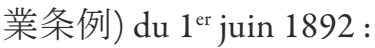

Lorsque les opérations de prospection ou d'extraction portent préjudice à l'intérêt général, il est possible d'annuler les autorisations et concessions relevant des juridictions respectives de l'inspecteur en chef des mines pour la prospection, et du ministère de l'Agriculture et du Commerce pour l'extraction.

第一九条:試掘若八採掘ノ事業公益二害アルトキハ試 掘二就テハ所轄鉱山監督署長採掘二就テハ農商務大臣既 二與ヘタル認可若ハ特許 7 取消スコト 7 得 ${ }^{28}$ 。

Qu'attend donc le gouvernement pour réagir devant la gravité d'une situation où les activités d'un individu portent préjudice à l'intérêt général (kōeki 公益) ? La réponse à cette interrogation de Tanaka, qu'il devra d'ailleurs solliciter par deux fois, n'arrive que le 18 mars $1897^{29}$, signée d'Enomoto et du ministre de l'Intérieur Kabayama Sukenori 樺山資紀 (1837-1922). Rappelons que des arrangements à l'amiable d'une durée de quatre ans ont déjà été négociés en 1892 entre Furukawa et les victimes de la pollution, qui avaient alors renoncé à toute

27. Cette interpellation s'intitule « Kōeki ni yūgai no kōgyō o teishi sezaru gi ni tsuki shitsumon-sho 》公益二有害ノ鉱業习停止セザル儀二付質問書 (Question sur la nécessité d'interrompre les activités minières portant préjudice à l'intérêt général), in TANAKA, 1989, pp. 53-58.

28. Le texte est cité dans Shōji \& Sugai 2014 [1984], p. 50.

29. Еломото \& Кавауама, 1971 [1938]. 


\section{CIPANGO}

216 Cahiers d'études japonaises n²3

forme de protestation en échange d'une indemnisation financière. S'agissant d'accords de droit civil (minji 民事), ni le gouvernement, ni les autorités du département, ni celles du district n'en sont partie prenante. Il conviendra de noter l'insistance des deux ministres sur la dimension privée dans laquelle la résolution du conflit a jusqu'alors été laissée. Pas un mot de leur part, toutefois, quant à la durée limitée de ces arrangements à l'amiable, en l'occurrence échus depuis 1896. Par ailleurs, les ministres affirment que la pollution est un phénomène récurrent, qui dépasse le seul cas d'Ashio, et que ces désagréments ne manqueront pas de se reproduire ailleurs. Ils soulignent l'importance du développement de l'industrie minière pour l'économie du pays, et concluent que « des mesures appropriées devront être prises afin d'adopter des politiques tenant compte des confrontations ultérieures entre industrie minière et agriculture ${ }^{30}$. 》 Une remarque qui prouve en soi la reconnaissance par le gouvernement d'une divergence d'intérêts entre les deux secteurs. La problématique d'Ashio se voit ainsi relativisé par les enjeux, bien plus larges, du développement industriel du pays.

Dans leur missive, les deux ministres évoquent également les travaux préventifs entrepris par Furukawa suite à l'arrêté de décembre, ce qui leur permet d'éviter de se prononcer sur l'éventuelle violation de l'article 19 de la loi sur les activités minières, puisqu'il leur faut officiellement attendre la mise en service des installations afin de juger de leur efficacité. Cette « réponse » (tōben 答辯) ne déroge finalement pas au refus d'implication manifesté jusque-là par le gouvernement. Les dispositions prises par ses soins à la fin du mois de décembre 1896 lui auront donc aussi bien permis de gagner du temps que d'apaiser momentanément la colère des victimes et de l'opinion publique. Mais la situation commence à évoluer.

30. Ibid., p. 178. 


\section{La Commission d'enquête de 1897}

Le gouvernement institue en effet une nouvelle Commission d'enquête le 24 mars $^{31}$, quelques jours seulement après sa réponse à la Diète. La protestation est à présent suffisamment organisée pour maintenir une pression sur les autorités, notamment grâce à une « stratégie personnelle ${ }^{32}$ » faisant directement appel à des personnalités proches des cercles du pouvoir. Le conservateur et ancien ministre de l'Agriculture Tani Tateki 谷干城 (1837-1911) visitera la région, y entraînant le ministre en charge Enomoto, qui se rend sur place le 23 mars. Ce dernier, frappé par la gravité et l'étendue des dommages, bien plus lourds que décrit dans les rapports officiels, rentre immédiatement à la capitale et persuade le ministre des Affaires étrangères, Ōkuma Shigenobu 大隈重信 (1838-1922), de la nécessité d'une intervention. Les événements se précipitent : une deuxième marche de protestation est organisée le même jour. Plusieurs milliers de manifestants se dirigent vers Tōkyō ; leur destination, toutefois, n’est pas le ministère de l'Agriculture et du Commerce, mais le siège du ministère de la Maison impériale ${ }^{33}$, qu'ils ne parviendront pas à atteindre. La perspective d'une implication de la famille impériale contribue aussi certainement à faire réagir le gouvernement : le 24 mars 1897, soit le lendemain de la visite d'Enomoto, le Cabinet se réunit en séance extraordinaire et institue la Commission d'enquête, conformément à la promesse faite à la population locale. Après la démission d'Enomoto, le 29 mars de la même année, c'est Ōkuma qui lui succède à la tête du ministère de l'Agriculture et du Commerce, cumulant ainsi deux portefeuilles.

31. Autrement nommée « Première commission d'enquête sur la pollution minière » (Daiichiji kōdoku chōsa iinkai 第一次鉱毒調査会委員). Nous avons toutefois évoqué supra l'existence d'une précédente Commission, spéciale, qui ne dura que trois jours (instituée le 22 décembre 1896, elle remit son rapport le 25). Cette Commission spéciale de 1896 était interne au ministère de l'Agriculture et du Commerce, tandis que celle de 1897 est interministérielle.

32. L'expression est de Notehelfer, 1975, p. 372.

33. Le Kunaishō 宮内省, instauré en 1869 afin d'administrer les affaires de la maison impériale et de l'aristocratie. 
La Commission, dont le rôle demeure consultatif, se compose aussi bien de spécialistes que des directeurs des bureaux concernés par l'affaire : on retrouve ainsi Gotō Shinpei ${ }^{34}$, responsable du bureau de l'Hygiène du ministère de l'Intérieur, et Megata Tanetarō, à la tête du bureau des Impôts du ministère des Finances, sous la présidence de Kōmuchi Tomotsune, lui-même directeur du bureau législatif du Cabinet ${ }^{35}$. Sur les quatorze membres désignés entre le 24 et le 26 mars, bon nombre sont spécialisés dans l'agronomie, tels Sakano Hatsujirō, qui avait été dépêché sur place à l'hiver 1896, ou Nagaoka Muneyoshi. La Commission s'élargit une première fois le 12 avril avec l'intégration de Koizuka Ryū, directeur du bureau des Mines. Elle est complétée le 10 mai par un expert supplémentaire, Irisawa Tatsukichi ${ }^{36}$, alors professeur assistant à la Faculté de médecine de l'université impériale de Tōkyō, pour comprendre au final seize membres ${ }^{37}$.

34. Médecin de formation, à la tête du bureau de l'Hygiène depuis 1892, il sera ensuite directeur du bureau des Affaires civiles du gouvernement colonial à Taïwan (1898) et occupera ultérieurement plusieurs postes de ministre.

35. Le Hōseikyoku 法制局, qui dépend directement du Premier ministre, rédige des rapports sur les délibérations du gouvernement, des projets et des enquêtes concernant les fonctionnaires et les organismes administratifs. Il s'exprime sur l'élaboration des lois ou des décrets, ainsi que sur leur mise en vigueur, leur application, leur abrogation ou leur révision. Selon la Constitution de 1889 , le directeur du bureau appartient à l'état-major du Cabinet, au même titre que le secrétaire en chef, et joue un rôle proéminent en tant que haut responsable des questions juridiques.

36. Spécialiste de médecine interne et historien de cette discipline. Diplômé de l'université impériale (Tōkyō) en 1889, il étudie à Strasbourg et Berlin de 1890 à 1894. À son retour au Japon, il ouvre sa propre clinique. Dès 1895, il est professeur assistant à la Faculté de médecine de son université d'origine et passera professeur en 1901. Il sera à plusieurs reprises médecin attitré du ministère de la Maison impériale avant d'en devenir le médecin en chef en 1924.

37. Le total diffère selon les sources. Dix-neuf est le nombre maximal qui inclut encore Shiga Shigetaka 志賀重昂 (1863-1927), Shiga Taizan 志賀泰山 (1854-1934) et Shirasawa Yasumi 白沢保美 (1868-1947). Tous trois sont nommés le 13 octobre 1897. Voir Konishi, 1989. 


\section{TABLEAU 1. LA COMMISSION INTERMINISTÉRIELLE DE 1897}

\begin{tabular}{|c|c|c|}
\hline \multicolumn{3}{|c|}{$\begin{array}{l}\text { COMMISSION INTERMINISTÉRIELLE (24 mars-27 novembre 1897) } \\
\text { Ashio dōzan kōdoku jiken chōsa iinkai 足尾銅山鉱毒事件調査委員会 }\end{array}$} \\
\hline Commissaire & Date de nomination & Fonction \\
\hline $\begin{array}{c}\text { FURUICHI Kōi/ } \\
\text { Kimitake } \\
\text { 古市公威 } \\
\text { (1854-1934) }\end{array}$ & 24 mars & $\begin{array}{c}\text { Ingénieur en chef des Travaux publics } \\
\text { (Doboku gikan 土木技監) } \\
\text { du ministère de l'Intérieur } \\
\text { (Naimushō 内務省) }\end{array}$ \\
\hline $\begin{array}{l}\text { GOTŌ Shinpei } \\
\text { 後藤新平 }(1857-1929)\end{array}$ & 24 mars & $\begin{array}{l}\text { Directeur du bureau de l'Hygiène } \\
\text { (Eisei kyokuchō 衛生局長) } \\
\text { du ministère de l'Intérieur }\end{array}$ \\
\hline $\begin{array}{l}\text { HAYAKAWA Tetsuji } \\
\text { 早川鉄治 (?-1941) }\end{array}$ & 24 mars & $\begin{array}{c}\text { Secrétaire (daijin hishokan 大臣秘書 } \\
\text { 官) du ministère de l'Agriculture } \\
\text { et du Commerce } \\
\text { (Nōshōmushō 農商務省) }\end{array}$ \\
\hline $\begin{array}{l}\text { HOSOI Iwaya } \\
\text { 細井岩弥 }(1862-1937)\end{array}$ & 24 mars & $\begin{array}{l}\text { Ingénieur (gishi 技師) du ministère de } \\
\text { l’Agriculture et du Commerce }\end{array}$ \\
\hline $\begin{array}{l}\text { IRISAWA Tatsukichi } \\
\text { 入沢達吉 (1865-1938) }\end{array}$ & $10 \mathrm{mai}$ & $\begin{array}{c}\text { Professeur assistant (jokyōjū 助教授) } \\
\text { de la faculté de médecine } \\
\text { (Ika daigaku 医科大学) }\end{array}$ \\
\hline $\begin{array}{l}\text { KODERA Fujisarō } \\
\text { 小寺房次郎 (1870-1949) }\end{array}$ & 24 mars & $\begin{array}{l}\text { Ingénieur du ministère de l'Agriculture } \\
\text { et du Commerce }\end{array}$ \\
\hline $\begin{array}{l}\text { KOIZUKA Ryū } \\
\text { 肥塚龍 }(1848-1920)\end{array}$ & 12 avril & $\begin{array}{c}\text { Directeur du bureau des Mines } \\
\text { (Kōzan kyokuchō 鉱山局長) } \\
\text { du ministère de l'Agriculture et du } \\
\text { Commerce }\end{array}$ \\
\hline $\begin{array}{l}\text { KŌMUCHI Tomotsune } \\
\text { 神鞭知常 (1848-1905) }\end{array}$ & $\begin{array}{c}24 \text { mars } \\
\text { (Président) }\end{array}$ & $\begin{array}{c}\text { Directeur } \\
\text { du bureau législatif du Cabinet } \\
\text { (Hōsei-kyoku chōkan 法制局長官) }\end{array}$ \\
\hline $\begin{array}{c}\text { KOTŌ Bunjirō } \\
\text { 小藤文次郎 }(1856-1935)\end{array}$ & 26 mars & $\begin{array}{c}\text { Professeur (kyojju 教授) } \\
\text { à la faculté de sciences naturelles } \\
\text { (Rika daigaku 理科大学) }\end{array}$ \\
\hline
\end{tabular}




\section{CIPANGO}

\begin{tabular}{|c|c|c|}
\hline $\begin{array}{l}\text { MEGATA Tanetarō } \\
\text { 目賀田種太郎 } \\
(1853-1926)\end{array}$ & 24 mars & $\begin{array}{c}\text { Directeur du bureau des Impôts } \\
\text { (Shuzei kyokuchō 主税局長) } \\
\text { du ministère des Finances } \\
\text { (Ōkurashō 大蔵省) }\end{array}$ \\
\hline $\begin{array}{l}\text { NAGAOKA Muneyoshi } \\
\text { 長岡宗好 (1866-1907) }\end{array}$ & 26 mars & $\begin{array}{c}\text { Professeur assistant à la Faculté } \\
\text { d'agronomie } \\
\text { (Nōka daigaku 農科大学) }\end{array}$ \\
\hline $\begin{array}{l}\text { ODA Makoto } \\
\text { 織田一(1864-1914) }\end{array}$ & 26 mars & $\begin{array}{l}\text { Conseiller (sanjikan 参事官) du } \\
\text { ministère de l'Agriculture et du } \\
\text { Commerce }\end{array}$ \\
\hline $\begin{array}{l}\text { SAKANO Hatsujirō } \\
\text { 坂野初次郎 (1867-1903) }\end{array}$ & 24 mars & $\begin{array}{l}\text { Ingénieur du laboratoire expérimental } \\
\text { d'agriculture } \\
\text { (Nōji jikenjō 農事試験場) }\end{array}$ \\
\hline $\begin{array}{c}\text { TSUBOI Jirō } \\
\text { 坪井次郎 (1863-1903) }\end{array}$ & 26 mars & Professeur de la faculté de médecine \\
\hline $\begin{array}{c}\text { WADA Kunijirō } \\
\text { 和田国次郎 (1866-1941) }\end{array}$ & 24 mars & $\begin{array}{l}\text { Ingénieur du ministère de l'Agriculture } \\
\text { et du Commerce }\end{array}$ \\
\hline $\begin{array}{l}\text { WATANABE Wataru } \\
\text { 渡辺渡 (1857-1919) }\end{array}$ & 26 mars & $\begin{array}{c}\text { Ingénieur affilié au bureau } \\
\text { des Affaires impériales } \\
\text { (Hishoku gōryō kyoku 非職御料局) }\end{array}$ \\
\hline
\end{tabular}

\section{Fermer la mine ? L'enjeu au cœur des débats}

Après plusieurs inspections, la Commission commence à délibérer le 13 avril en vue de remettre ses recommandations au gouvernement. Le président Kōmuchi suggère d'emblée la possibilité d'interrompre en partie ou totalement les activités de la mine et de ne les reprendre qu'à condition de réaliser des travaux de prévention, puisqu'il s'agit tout de même d'une « production nationale d'importance » (taisetsu na kokusan 大切ナ国産) ${ }^{38}$. Ce point attise les oppositions. Voici le texte proposé : « une interruption temporaire de tout

38. « Ashio chōsa sokkiroku », 1980, p. 656. 
ou partie des activités de la mine d'Ashio [et] l'étude d'un moyen garantissant la maintenance complète et durable des ouvrages préventifs contre la pollution ${ }^{39}$. » Mais faut-il commencer par suspendre l'exploitation, ou exiger en premier lieu de l'opérateur qu'il prenne des dispositions ? La suspension, voire la fermeture, sont loin de faire l'unanimité. Un débat s'engage autour de la loi sur les activités minières, et notamment de son article 59. Celui-ci stipule :

En cas de risque ou lorsqu'un préjudice à l'intérêt général est constaté, le chef de la surveillance des mines ordonne à l'exploitant d'y parer ou d'interrompre les activités minières.

第五九条:鉱業上ニ危険ノ盧レアリ又ハ公益习害スコ 認ムルトキハ所轄鉱山監督署長八鉱業人二其ノ予防习命 シ又八鉱業习停止スヘシ40。

L'article ne précise pas exactement laquelle des deux mesures envisagées est prioritaire, offrant une marge à l'interprétation. Watanabe Wataru défend la première et suggère d'établir une date butoir pour les travaux ; si l'exploitant n'a pas observé les directives passé ce délai, les activités pourront être interrompues ${ }^{41}$. Des mesures ayant déjà été lancées suite à l'arrêté de décembre $1896^{42}$, il considère qu'une interruption serait «punitive » (chōbatsuteki 懲誩的) ${ }^{43}$, et insiste sur la nécessité de ne plus songer uniquement à l'agriculture, mais de prendre par ailleurs en considération les besoins de l'industrie minière, à l'image de l'Europe : «De nos jours, on ne peut plus dire que l'agriculture constitue la base de la nation ${ }^{44}$. » Une critique de l'agrarisme visant clairement Kōmuchi, proche de ce courant, et qui lui répond : « L'agriculture japonaise est probablement très
39. Ibid., p. 692.
40. Cité dans Shōji \& Sugai, 2014 [1984], p. 50.
41. « Ashio chōsa sokkiroku », 1980, p. 693.
42. Ibid., p. 694.
43. Ibid., p. 694-695.
44. Ibid., p. 695. 


\section{CIPANGO}

222 Cahiers d'études japonaises $n^{\circ} 23$

différente de l'européenne. Vous affirmez que l'agriculture n'est plus la base de la nation, mais dans les faits, c'est largement le $\operatorname{cas}^{45}$. $\gg$ Se dévoile ainsi, d'emblée, une profonde fracture idéologique au sein de la Commission. Lors de la troisième séance, le 15 avril, Nagaoka rappelle les inquiétudes de la population et propose des interventions techniques, notamment des améliorations quant au lavage du minerai, phase des plus polluantes. Celles-ci, toutefois, n'influenceraient que peu le fonctionnement de la mine. Furuichi, de son côté, est convaincu qu'il est possible de se prémunir suffisamment contre la pollution sans fermer la mine ${ }^{46}$. Sakano partage pour sa part le point de vue de Nagaoka, et insiste :

[L]aisser la situation en l'état jusqu'à la réalisation des travaux préventifs serait manquer de bienveillance et de sens de la justice. À plus forte raison si le poison continue à se déverser, quand bien même il n'y aurait aucun lien [entre les rejets toxiques et les dégâts sur les cultures $]^{47}$.

Nagaoka renchérit : «Watanabe ne parle que de la mine, sans la moindre considération pour l'agriculture ${ }^{48} \gg$, mais au moment du vote, c'est finalement la ligne prônée par Watanabe, avec le soutien de Gotō et Furuichi, qui est acceptée à la majorité (par huit voix sur les douze membres présents ce jour-là). Koizuka, pourtant compagnon de parti de Tanaka et signataire de l'interpellation présentée à la Diète le 26 février 1897, s'y est rallié. Le passage débattu prend finalement la forme suivante :

1. Déterminer une date pour l'étude et la mise en application de moyens garantissant la maintenance complète et durable des ouvrages préventifs contre la pollution minière et les fumées toxiques. En outre, si nécessaire, ordonner des inspections directes par les autorités, dont les coûts seront à la charge de l'exploitant de l'exploitation, ou interrompre les activités minières.

45. Ibid., p. 696.

46. Ibid., p. 705.

47. Ibid., p. 711.

48. Ibid., p. 713. 


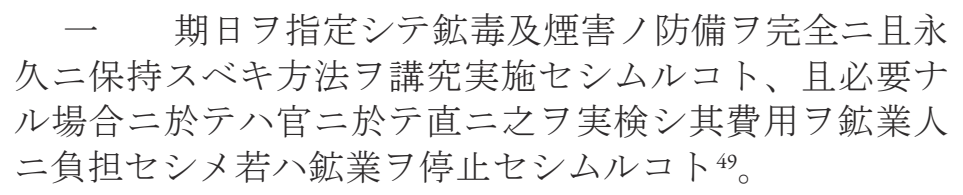

Dès le 15 avril, la possibilité de fermer la mine comme le demandent les protestataires s'éloigne donc considérablement. Cet enjeu essentiel sera à nouveau débattu dans un deuxième temps. Le 12 mai, lors de l'avant-dernière séance, Sakano et Nagaoka présentent une « motion d'urgence » (kinkyū dōgi 緊 急動議) motivée par de nouvelles analyses qui les ont confrontés «à une vérité impossible à taire ${ }^{50} \gg$. Lors d'analyses complémentaires effectuées sur place, les deux commissaires ont en effet constaté plusieurs complications : les eaux qui s'échappent des galeries abandonnées sont peu abondantes et faiblement acides, contrairement à celles provenant des galeries en activité. Les terrils, dont la quantité est bien supérieure aux estimations, sont en outre massivement déversés dans la rivière, au point d'en obscurcir complètement le fond. Comme la boue sablonneuse des étangs de sédimentation est jetée sur les berges, les éléments

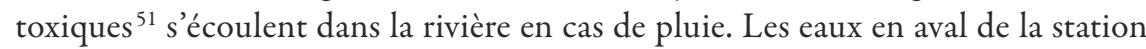
d'affinage de Kodaki 小滝 sont contaminées et fortement chargées de cuivre ${ }^{52}$. Tous ces faits ont été cachés par les responsables de la mine : Nagaoka et Sakano affirment s'être heurtés sur place à de flagrants signes d'obstruction et de dissimulation de leur part. Lorsqu'ils ont tenté de récolter des échantillons, « les salariés de la mine ont volontairement caché l'endroit d'où ils évacuent l'eau et en ont drastiquement réduit le débit ${ }^{53}$. » Au demeurant, l'injection de chaux dans l'évacuation des eaux usées, procédure pourtant préconisée dans l'arrêté

49. Ibid., p. 717.

50. Ibid., p. 758.

51. On utilise pour traiter le minerai plusieurs substances (arsenic, chlore, sulfate de cuivre, soufre), de même que des métaux lourds (cadmium, plomb, mercure, zinc).

52. Komatsu résume toutes ces observations ; nous avons retenu les plus saillantes. Komatsu, 2001, p. 346.

53. « Ashio chōsa sokkiroku », 1980, p. 779. 
de 1896, n’a nullement été appliquée. Puisque le manque de rigueur de l'exploitant est désormais évident, Nagaoka et Sakano demandent en conséquence l'interruption, partielle ou complète, des activités minières jusqu'à l'achèvement des installations de prévention.

Leur motion est violemment attaquée : Wada Kunijirō accuse Sakano de donner des exemples exagérés et ridicules ${ }^{54}$, Koizuka persiste à vanter l'efficacité des travaux de prévention, et Gotō considère qu'arrêter l'exploitation sur la base de simples suppositions serait injustifié ${ }^{55}$. La discussion s'emballe, mais lors du vote final, la résolution de Sakano et Nagaoka ne recueille que quatre voix favorables sur les douze membres présents. Si cette ultime tentative d'interrompre les activités minières échoue, bien que fondée sur des analyses récentes, elle déclenche néanmoins une réaction : au lendemain de cette réunion houleuse, soit le 13 mai, un deuxième arrêté est adressé à Furukawa. Ce dernier est sommé de prendre une série de dispositions concernant à la fois l'évacuation des débris, les appareils de dispersion automatique de chaux, le transfert et le stockage des déblais et sédiments, avec des indications précises sur les sites concernés et les différentes interventions à mener ${ }^{56}$. Puisque cet arrêté prend très exactement en compte les éléments soulevés la veille par Sakano et Nagaoka, on pourrait croire que le gouvernement en vient enfin à des mesures plus strictes. Ce texte est cependant très court (il ne compte que quatre points) et peu contraignant, analogue en ceci au premier arrêté de décembre 1896. Il faut, pour bien comprendre les enjeux du 12 mai, rappeler que l'arrivée dans la Commission du professeur Irisawa deux jours plus tôt ne doit rien au hasard. La dernière nomination, celle de Koizuka, remonte au mois précédent, plus exactement au 13 avril. Informé de la présence de Sakano et Nagaoka sur le terrain, le ministère de l'Agriculture et du Commerce manœuvre alors pour que la Commission ne rouvre pas la discussion sur la fermeture de la mine, et nomme Irisawa en urgence. Épaulé par Gotō, ce dernier jouera en effet un rôle déterminant lors de la dernière séance, qui entérine l'adoption de dispositions peu rigoureuses à l'encontre de Furukawa.

54. Ibid., p. 784.

55. Ibid., p. 778.

56. SōGYō HyaKUnENSHI HENSAN IINKAI, 1976, p. 167. 
Ainsi, le 18 mai, Irisawa, Gotō et Tsuboi Jirō, les trois commissaires avec une formation de médecin, présentent le compte-rendu de leurs recherches. À leur avis, la pollution n'a aucun effet direct sur le corps humain. Le même jour, ils adressent au Cabinet un rapport écrit (jōshinsho 上申書), dans lequel ils proposent de trouver un moyen pour prévenir la pollution des cours d'eau, tout en se montrant très réservés sur la nécessité de travaux de prévention. À plusieurs reprises, Gotō avait déjà exprimé sa ferme opposition à l'interruption de l'exploitation, et il confirme cette position lors de la séance du 12 mai. Le compte rendu (fukumeisho 復命書) qu'il remettra au Cabinet quelques mois plus tard, le 14 octobre 1897, reprend en partie son exposé du 18 mai.

Rappelons que les commissaires ne sont pas des experts indépendants, mais pour la plupart, des fonctionnaires, d'où l'importance de reconstituer l'argumentaire des défenseurs de la mine et de se pencher sur les éléments de preuve qu'ils fournissent. Prenons donc le « rapport d'enquête » personnel (chōsa hōkokusho 調査報告書) que Gotō joint à son compte-rendu d'octobre : les résultats qu'il y présente posent au moins deux problèmes ${ }^{57}$. Le premier concerne les échantillons prélevés pour les analyses (urine et selles, eau de puits). Ceux-ci avaient d'ailleurs déjà été remis en question par un article du Yomiuri shinbun 讀賣新聞 du mois d'avril. Intitulé « Les échantillons du directeur du bureau de l'Hygiène Gotō ne valent rien ${ }^{58} \gg$, cet article rapporte l'opinion d'un médecin diligentant des études sur les terres contaminées : les échantillons en question proviendraient selon lui de paysans des couches supérieures, qui se nourriraient d'aliments produits ailleurs ; leur état de santé ne serait donc pas représentatif. De même pour l'eau des puits, qui serait filtrée et purifiée avant consommation. Alors qu'il ne peut ignorer ces réserves, Gotō ne fournit pas de réponse, ni dans son exposé du 18 mai, ni dans le rapport remis en octobre.

Le second problème concerne l'affirmation selon laquelle la pollution ne porte pas d'atteinte directe à la santé humaine. Une donnée statistique vient

57. Ces contradictions sont soulevées par Konishi, qui démontre bien comment la question médicale est utilisée pour éviter la fermeture de la mine. Konishi, 1989, p. 773-776.

58. Yomiuri shinbun, 14 avril 1897. 
cependant contredire ce propos : le pourcentage de jeunes hommes des départements de Tochigi et de Gunma recalés à l'examen médical pour le service militaire. Comparés à la moyenne nationale, le taux d'échec des deux départements est pratiquement identique, voire légèrement inférieur au reste du pays. Mais si l'on observe la situation au niveau des districts et des villes, on remarque une nette différence dans les zones sinistrées, lesquelles présentent un taux plus élevé de refus. Lors de la dernière séance, le 18 mai, Gotō annonce être en train de rassembler du matériel afin d'élucider les raisons de cet échec à l'examen militaire, mais ne fournit pas de réponse claire.

Gotō s'appuie sur les recherches menées par Irisawa, qui fut détaché sur place suite au signalement des inspections de Sakano et Nagaoka ${ }^{59}$. Les résultats de l'assistant universitaire prennent donc un poids considérable. Il se trouve qu' Irisawa n'est pas dépourvu de liens avec la mine d'Ashio, puisqu' il est proche de Konda Bunjirō 昆田文次郎 (1862-1927), un ancien conseiller juridique engagé par la mine depuis le début de l'année 1897 en tant que vice-manager et chargé des relations avec les milieux politiques ${ }^{60}$. En choisissant Irisawa pour mener des analyses, le ministère de l'Agriculture et du Commerce sait d'ores et déjà qu'il ne recevra probablement pas de rapport accablant pour la mine.

Le ministère s'active aussi à d'autres niveaux. Le 26 avril, il institue en son sein une Commission restreinte réunissant six de ses fonctionnaires ${ }^{61}$ : le vice-ministre Ōishi Masami, président, Takahashi Takuya et Fujita Shirō, respectivement directeurs du bureau des Forêts et des Affaires agricoles, auxquels s'ajoutent trois membres de la Commission interministérielle (Koizuka, Oda Makoto et Hayakawa Tetsuji ${ }^{62}$. Le même jour, le ministère charge l'un de

59. Avant même sa nomination, Irisawa, qui a été approché dès la fin du mois d'avril, est détaché dans les terres contaminées, où il mène des analyses du 3 au 5 mai. Ibid., p. 777.

60. Ibid.

61. Kōdoku chōsa iinkai 鉱毒調査委員会 («Commission d'enquête sur la pollution minière », ci-après « Commission restreinte »). Voir le tableau 2.

62. Konishi, 1989, p. 785-786. Le fait que les trois membres siégeant dans les deux commissions soient tous favorables à la mine en dit long sur le véritable rôle joué par la Commission restreinte. Konishi revient sur cette question dans KonIsHi, $1990 \mathrm{~b}$. 
ses fonctionnaires, Hotta Rentarō 堀田連太郎 (1857-1915), de rédiger un projet de mesures à l'encontre de la mine. Ce texte deviendra le troisième arrêté, daté du 27 mai 1897. S'ils sont au courant de l'existence d'une Commission restreinte depuis le 28 avril $^{63}$, les membres de la Commission n'ont pas l'air de savoir que quelqu'un d'autre travaille parallèlement à la rédaction du futur arrêté. Hotta assiste à la dernière séance, celle du 18 mai, durant laquelle les commissaires l'interrogent pour savoir si sa version tient bien compte de leurs recommandations. Celui-ci se défend en affirmant que son texte « a été composé en fonction des décisions récentes de la Commission, en observe les grandes lignes et a été préparé sur leur modèle ; le détail des modalités relève déjà du ressort du ministre ${ }^{64}{ }$, signifiant aux membres de la Commission que la rédaction de l'arrêté ne relève pas (ou plus) de leur compétence. Le président Kōmuchi, en tout cas, ne semble pas apprécier ce changement : « si l'ébauche n'avait pas dû passer devant une réunion du Cabinet ou si elle n'était pas parvenue entre mes mains, j'aurais pu ne pas en avoir connaissance ${ }^{65} \gg$, remarque-t-il, estimant souhaitable de réunir en urgence les membres de la Commission.

63. Koizuka l'annonce le 28 avril. « Ashio chōsa sokkiroku », 1980, p. 729.

64. Ibid., p. 810.

65. Ibid., p. 810. 


\section{CIPANGO}

228 Cahiers d'études japonaises $n^{\circ} 23$

TABLEAU 2. LA COMMISSION RESTREINTE D’AVRIL 1897

\begin{tabular}{|c|c|c|c|}
\hline \multicolumn{4}{|c|}{$\begin{array}{c}\text { COMMISSION RESTREINTE (26 avril 1897) } \\
\text { Ködoku chōsa iinkai 鉱毒調査委員会 }\end{array}$} \\
\hline Commissaire & $\begin{array}{c}\text { Date de } \\
\text { nomination }\end{array}$ & Fonction & Notes \\
\hline $\begin{array}{l}\text { FUJITA Shirō } \\
\text { 藤田四郎 } \\
(1861-1934)\end{array}$ & 26 avril & $\begin{array}{c}\text { Directeur du bureau } \\
\text { des Affaires agricoles } \\
\text { (Nōmu kyokuchō 農務局長) } \\
\text { du ministère de l'Agriculture } \\
\text { et du Commerce }\end{array}$ & \\
\hline $\begin{array}{l}\text { HAYAKAWA } \\
\text { Tetsuji }\end{array}$ & 24 mars & Voir tableau 1 & $\begin{array}{c}\text { Membre de la } \\
\text { Commission } \\
\text { interministérielle }\end{array}$ \\
\hline $\begin{array}{l}\text { KOIZUKA } \\
\text { Ryū }\end{array}$ & 12 avril & Voir tableau 1 & $\begin{array}{c}\text { Membre de la } \\
\text { Commission } \\
\text { interministérielle }\end{array}$ \\
\hline ODA Makoto & 26 mars & Voir tableau 1 & $\begin{array}{l}\text { Membre de la } \\
\text { Commission } \\
\text { interministérielle }\end{array}$ \\
\hline $\begin{array}{l}\text { ŌISHI Masami } \\
\text { 大石正巳 } \\
(1855-1935)\end{array}$ & 26 avril & $\begin{array}{c}\text { Vice-ministre (jikan 次官) } \\
\text { du ministère de l'Agriculture } \\
\text { et du Commerce }\end{array}$ & $\begin{array}{l}\text { Président de la } \\
\text { Commission } \\
\text { restreinte }\end{array}$ \\
\hline $\begin{array}{l}\text { TAKAHASHI } \\
\text { Takuya } \\
\text { 高橋玩也 } \\
(1848-1935)\end{array}$ & 26 avril & $\begin{array}{l}\text { Directeur du bureau des Forêts } \\
\text { (Sanrin kyokuchō 山林局長) } \\
\text { du ministère de l'Agriculture } \\
\text { et du Commerce }\end{array}$ & \\
\hline
\end{tabular}

En fin de compte, l'arrêté du gouvernement ne sera donc pas rédigé par la Commission interministérielle. Si les membres de la Commission ont ne serait-ce qu'eu la possibilité de le lire, c'est bien parce que Kōmuchi avait eu l'ébauche entre les mains à titre de directeur du bureau législatif, et surtout parce qu'il décida d'en discuter durant la séance. On ne pourra que s'étonner du fait que la Commission 
spécialement mise sur pied n'ait pas eu de rôle à jouer dans l'élaboration d'un tel document, ni au moins son mot à dire. Non content de créer une Commission restreinte faisant doublon avec la Commission interministérielle, le ministère de l'Agriculture et du Commerce dépossède celle-ci de son pouvoir d'action et la maintient dans l'ignorance des dispositions finales.

\section{Des dédommagements à la charge de l'État}

En sus de l'éventuelle fermeture de la mine, la seconde grande question discutée par la Commission est celle des dédommagements, thème essentiel sur lequel porteront les sessions à partir du $26 \mathrm{avril}^{66}$. Quelques jours auparavant, le 21 avril, une partie des protestataires s'était réunie et avait fait savoir sa détermination à obtenir justice. Ils déclarent ainsi au Journal de Tochigi (Tochigi shinbun 杤木新聞) : 《Si les discussions de la Commission d'enquête devaient par hasard, comme le prétendent les rumeurs, ne pas aboutir à la fermeture de la mine, alors toutes les victimes tenteront de monter à la capitale, bien résolues à mourir ${ }^{67}$. » Selon le journal, les volontaires du district d'Ōra 邑楽 (Gunma) avaient d'ores et déjà constitué un «commando de choc » (kesshitai 決死隊) de plus de cinquante personnes, tandis que des mouvements similaires se dessinaient à Ashikaga 足利 et Aso 安蘇 (Tochigi). C'est soumis à cette pression que les membres de la Commission discutent des éventuelles réparations à accorder, envisageant assez rapidement des « exemptions fiscales 》 (menso shobun 免租処分), possibilité prévue par la « législation sur l'impôt foncier 》 (chiso jōrei 地租条例 $)^{68}$.

66. Ce sujet occupe une bonne partie des quatrième à septième séances, celles du 26 , 28 avril, 3 et 7 mai. Il est relativement peu traité dans la littérature secondaire, sauf par Komatsu, qui fournit quelques indications à ce propos. Komatsu, 2001, p. 344-345, 67. Dans l'édition du 25 avril : « kōdoku chōsa iin no kekka ni shite mangaichi uwasa no gotoku hiteishi to keshitaran ni wa higaimin ichidō shukkyō shite kesshi no daiundōo kokoroshimuru koto 》「鉱毒調査委員の結果にして万一噂の如く非停止と決し たらんには被害民一同出京して決死の大運動を試むる事」, p. 344.

68. La 《 Loi sur l'impôt foncier 》 (chiso jōrei 地租条例) avait succédé en 1884 à celle de 1873 (chiso kaisei 地租改正), parfois aussi appelée chiso kaisei jörei 地租改正条例. 


\section{CIPANGO \\ 230 Cahiers d'études japonaises $n^{\circ} 23$}

Megata, directeur du bureau des Impôts, rappelle que le ministère des Finances entre en matière pour des allégements fiscaux lorsque des terrains agricoles deviennent soit 《 terres incultivables » (arechi $i$ 荒地) suite à un désastre naturel, soit impropres à la culture et/ou stériles ${ }^{69}$. Ainsi, pour qu'un propriétaire puisse bénéficier d'exemptions, il faut que son terrain soit reconnu comme tel. Est alors appliqué l'article 20 de la loi sur l'impôt foncier, lequel stipule : « À partir de l'année du sinistre et pendant dix ans, les terres incultivables bénéficient d'une exemption fiscale. À la fin de cette période, la valeur originelle de la terre sera restaurée $^{70}$. » Le débat se concentre sur la question du déclassement des terrains. Selon les explications de Megata, « on considère impropre à la culture un terrain modifié par un désastre naturel, dont la surface a par exemple diminué, ou a été recouverte par des pierres et du sable ${ }^{71}$. »

Kōmuchi résume comme suit les deux conditions sine qua non pour qu'un terrain soit déclassé : il faut « que les dommages soient provoqués par un désastre d'origine naturelle et que la configuration du sol ait été modifiée ${ }^{72}$. » Or, selon Megata, ces critères ne sont pas remplis en l'espèce ${ }^{73}$. Plusieurs membres s'alignent sur cette position, puisque la loi sur l'impôt foncier ne prévoit des exemptions que pour les dégâts d'origine naturelle, ce qui n'est pas le cas de la pollution qui les préoccupe.

Lors de la séance suivante, le 28 avril, Oda propose d'étendre le champ d'application de la loi : puisqu'on accorde des exemptions fiscales lorsque des pierres se déposent dans les rizières, pourquoi ne pas faire la même chose lorsque la pollution minière empêche en l'occurrence la récolte ${ }^{74}$ ? Dans ce passage, la posture d'Oda est ambigüe : aimerait-il que la pollution minière soit considérée

69. Dans un autre registre, le terme arechi désigne également les terres en friche.

70. «Arechi wa sono higai no toshi yori jünen inai menso nenki o sadame nenkigatsu ni itari genchika ofukusu 》「荒地八其被害ノ年ヨリ十年以内免租年期 至リ原地価二復ス」, cité dans AKAISHI, 2009, p. 11.

71. « Ashio chōsa sokkiroku », 1980, p. 718.

72. Ibid., p. 733.

73. Ibid., p. 720

74. Ibid., p. 732. 
comme une catastrophe naturelle - ce qui évacuerait en quelque sorte la question de l'origine du sinistre - ou demande-t-il à ce que les terres contaminées soient simplement déclassées et bénéficient ainsi d'une exemption fiscale ? Plus loin, celui-ci invite expressément à modifier la loi sur l'impôt foncier ${ }^{75}$, orientant à nouveau le débat vers cette possibilité, qui avait déjà été balayée par Megata ${ }^{76}$. Le 3 mai, la modification est remise à l'ordre du jour, cette fois par Nagaoka, dont l'argumentation est très simple : la loi sur l'impôt foncier ne pouvait pas prévoir les conséquences d'une éventuelle pollution pour avoir été promulguée avant l'apparition d'un phénomène de cette ampleur ${ }^{77}$. Il propose même d'aller plus loin et d'adopter, compte tenu des circonstances exceptionnelles, un 《 décret impérial urgent » (kinkyū chokurei 緊急勅令). Le secours aux zones contaminées pourra ainsi être plus rapide, tandis que la procédure de modification de la loi sur l'impôt foncier suivra son rythme et sera présentée à la Diète lors de la session de novembre ${ }^{78}$. Même si Oda souscrit à cette proposition, Megata continue de considérer que l'affaire en cours n'entre pas dans le champ d'application de la loi ${ }^{79}$. Koizuka, de son côté, s'oppose aussi à une modification, tout en précisant :

Quant à savoir s'il s'agit d'une action humaine inévitable, la pollution minière se distingue de ce qui jaillit des vallées ou des montagnes à l'improviste, si l'on en croit les explications des spécialistes. La pollution qui provient des exploitations minières est d'origine humaine, et comme le confirment les progrès de l'enquête ces derniers jours, on doit pouvoir s'en prémunir.

75. Ibid., p. 732-733.

76. Ibid., p. 723.

77. Ibid., p. 737.

78. Ibid., p. 737-738. Les similitudes entre le point de vue de Nagaoka et celui défendu par Tanaka à la Diète ne manquent pas d'étonner : pour Komatsu, Tanaka se serait inspiré de Nagaoka. Komatsu, 2001, p. 345.

79. « Ashio chōsa sokkiroku », 1980, p. 740. 
232 Cahiers d'études japonaises n²3

鉱誌卜云フ事八専門家ノ此問中カラノ説习聴イテ見ル
ノ人為デ防ギ得ベカラザモノ゙アルカト云フ事ニ就
テハ不意ニ山カラ噴キ出ス谿カラ噴キ出スト云フコトハ
別段ニシテ、鉱業习営ンデ居ルト云フ場所ノ鉱毒ト云フ
モノハ人為デ防ギ得ベキモノデアルト云フコトラバ此問
中カラノ調査ノ進行ニ依ツテ私ハモウ認メテ居ルデゴザ
イマス ${ }^{80}$

Megata, revenant sur l'origine de la pollution, suggère de procéder différemment : « Je pense que si les dommages causés à la nature ont une origine, il n'y a d'autre solution que d'identifier cette origine et de porter plainte ${ }^{81}$. » S'il est possible de déterminer l'origine des dégâts causés aux terres agricoles et de les expliquer par la pollution minière, la question relève en d'autres termes du droit civil, et la voie à suivre pour régler la dispute, de l'action en justice.

On pourra certes s'étonner que la responsabilité de l'industrie minière ne soit pratiquement pas abordée. Elle fut, en réalité, précédemment évacuée. Le 14 avril, la Commission avait discuté de l'origine des dégâts et des nuisances : était-ce le site en général (vieilles galeries désaffectées comprises) et, par là même, le fait des exploitants précédents ? Ou l'exploitation une fois prise en main par Furukawa? La mine d'Ashio était-elle exclusivement concernée, ou se trouvait-il d'autres sources éventuelles, à l'instar des rejets de l'industrie textile du district de Kiryū 桐生? Bien que les analyses de Nagaoka aient démontré qu'aucune autre source potentielle ne contenait de cuivre, la majorité des commissaires accepte de ne pas imputer les dégâts à la seule mine.

On constate une grande prudence, pour ne pas dire de la complaisance, à l'endroit du responsable. Dans les procès-verbaux, le nom de l'entreprise minière est soigneusement tu. Le but semble bien être de relativiser, voire de contester, la responsabilité de Furukawa dans l'affaire. Cette tendance à minimiser l'implication de la mine est perceptible dès le début, par exemple chez Gotō. Interrogé le 4 avril à son retour d'une tournée d'inspection dans les zones

80. Ibid., p. 748.
81. Ibid., p. 720. 
contaminées, il avait estimé très prématuré d'attribuer aux seules activités minières la cause du spectacle désolant auquel il avait été confronté ${ }^{82}$. Quand Megata, refusant la modification de la loi sur l'impôt foncier pour soulager les propriétaires des terres contaminées (et indirectement, les dédommager), affirme qu'il leur faut trouver les responsables et porter plainte, ce n'est pas sans une certaine hypocrisie de sa part, puisque la majorité de la Commission s'est déjà prononcée en faveur d'une formulation minimisant la responsabilité de l'exploitant.

Cet aspect mériterait qu'on s'y attarde. Il ne nous est pas clairement apparu pour quelles raisons les opposants à la mine n'avaient pas fait le choix d'une poursuite judiciaire. La difficulté à faire établir de manière définitive la responsabilité directe de l'industrie Furukawa, et ce en dépit de multiples analyses scientifiques, les en a très probablement dissuadés. En outre, en admettant qu'il ait pu fournir une base juridique suffisante, le Code civil venait tout juste d'être promulgué, au début de 1896, et n'entrera en vigueur que le 16 juillet 1898.

Nagaoka réagit vivement contre ce qu'il considère être une démission vis-àvis de la population : «Il faudrait vraiment manquer de considération pour se limiter à dire qu'il n'existe pas de législation, reléguer le problème au droit civil, les laisser se débrouiller, s’en débarrasser. » Et de demander : « Cela ne signifie-t-il pas abandonner une partie de la population ${ }^{83}$ ? »

Bien que cet avis soit partagé par Kōmuchi, la proposition faite par Oda d'accorder des exemptions fiscales pour les terres contaminées ne parvient pas à réunir la majorité ; elle est finalement rejetée le 3 mai. Ce point est cependant réexaminé lors de la séance du 7 mai, où l'on adopte, à la majorité de cinq membres sur les huit présents, un « projet de résolution spéciale » (tokubetsu shobun hōan 特別処分法案) distinguant trois sortes de terres affectées par la pollution:

1. les terres touchées par l'effondrement des digues dû aux inondations ;

2. les terres qui ont été inondées ;

3. les terres affectées par l'eau d'irrigation.

82. Journal Mainichi (Mainichi shinbun 毎日新聞) du 7 avril 1897, cité par KonisHI, 1989, p. 770 .

83. « Ashio chōsa sokkiroku », 1980, p. 750. 
Les premières et deuxièmes sont considérées comme des « terres incultivables », et exemptées selon l'article 20 de la loi sur l'impôt foncier. Pour le troisième type, la solution devra être trouvée sur le plan civil. Les procès-verbaux précisent toutefois que les avis divergent quant à ce dernier point : certains commissaires pensent qu' il faudrait trouver une solution analogue, mais au niveau de la législation fiscale ${ }^{84}$.

La majorité de la Commission a ainsi privilégié l'adoption d'une résolution spéciale à une modification plus profonde de la législation en vigueur, pourtant lacunaire. On peut considérer que le rapport de force entre défenseurs de la mine et partisans de strictes mesures à son encontre se solde par une victoire en demiteinte pour ces derniers. Comparé à la question de l'éventuelle fermeture de la mine, celle des dédommagements ne semble pas susciter une guerre de positions aussi forte. S'il est vrai que la proposition d'Oda a permis de déclasser en « terres incultivables » une partie des surfaces contaminées, ce compromis ne peut vraiment être considéré comme un progrès pour les victimes. Cette solution aura en réalité des conséquences dramatiques, puisque les exemptions fiscales n'équivalent pas pour autant à des compensations financières. Au lendemain du 18 mai, dernier jour de discussion pour la Commission, le gouvernement annonce qu'il ne se joindra pas aux éventuelles négociations entre l'industrie minière et la population.

\section{L'arrêté du 27 mai 1897}

À la fin des travaux de la Commission, le gouvernement prend des dispositions : le 27 mai 1897, le ministère de l'Agriculture et du Commerce adresse une première 《directive administrative 》 (kunrei 訓令) au bureau des Forêts, une seconde aux départements de Tochigi et Gunma, et un arrêté à l'exploitant de la mine, le troisième après ceux de décembre 1896 et du 13 mai 1897. Fort de trentehuit points, ce dernier document précise les travaux à entreprendre : bassins de sédimentation et de filtrage des eaux usées, équipements de contrôle des émissions de la raffinerie (sulfure), et espaces sécurisés de stockage pour les débris de minerai

84. Ibid., p. 635-636. 
et déblais, en prévention des éboulements comme de la contamination du réseau hydrique. Le délai imposé à Furukawa est strict : les opérations doivent débuter dans les sept jours pour être achevées sous trente à cent cinquante jours selon les ouvrages, sous peine de fermeture immédiate de l'exploitation. Cette décision est généralement considérée comme drastique, notamment en raison des dates butoir rapprochées et de la menace de fermeture pesant sur les travaux ${ }^{85}$. À la lumière des discussions sommairement retracées plus haut, on reste toutefois perplexe : le gouvernement aurait-il voulu se montrer plus sévère que ce que préconisaient les commissaires ? On peut raisonnablement en douter, surtout devant la comparaison détaillée que Konishi établit entre la première version de l'arrêté et le texte finalement signé par le Cabinet. Les travaux complexes envisagés au début se transforment à plusieurs reprises en interventions bien plus simples ; quant au texte en lui-même, il demeure suffisamment vague pour ne pas représenter de véritable contrainte, laissant incidemment à Furukawa une marge de manœuvre appréciable. Le point 32, qui spécifie les délais, a certes été durci et accompagné d'une menace de suspension immédiate, mais l'impression de grande fermeté qui s'en dégage est somme toute trompeuse. Furukawa dépensa des montants considérables - près de la moitié de la valeur totale de sa production annuelle de cuivre - pour réaliser une partie de ces travaux, qui donneront d'ailleurs lieu à des innovations technologiques substantielles ${ }^{86}$. Les autorités n'exerceront néanmoins aucune surveillance ni sur l'exécution des mesures, ni sur leur efficacité, ni même sur le respect des délais ${ }^{87}$. L'ampleur du programme et des sommes investies marquèrent peut-être les esprits, mais n'aidèrent pas à combattre efficacement la pollution, qui ne disparut pas pour autant.

Parallèlement, toujours le 27 mai, le ministère des Finances adresse aux 《fonctionnaires fiscaux 》 (zeimu kanri 税務官吏) des directives concernant l'exonération d'une partie des terrains contaminés. L'application de cette

85. Konishi recense les différentes interprétations de ce point proposées dans les années 1970 et 1980. Konishi, 1989, p. 747-754,

86. Sur ces questions, voir notamment MuraKami, 2006, p. 210-216.

87. Sur la mise en place des travaux préventifs ainsi que sur les délais, voir Konishi, 1990a. 


\section{CIPANGO \\ 236 Cahiers d'études japonaises $n^{\circ} 23$}

mesure, toutefois, s'avère extrêmement compliquée, puisque les enquêtes sur les dégâts ne progressent que lentement. Non seulement les propriétaires et les fonctionnaires ne s'accordent pas sur la désignation des parcelles déclassées, mais la communication entre les autorités départementales et centrales est difficile, ainsi qu'entre le ministère de l'Agriculture et du Commerce et celui des Finances. Les premières exonérations, qui ne seront décidées que le 2 mai $1898^{88}$, comportent un écueil supplémentaire : dans un système où le droit de vote est censitaire, elles équivalent à une perte de droits pour les bénéficiaires, ainsi privés de la possibilité d'élire leurs représentants, seuls à même de plaider leur cause. Les impôts locaux étant de surcroît associés à l'impôt national, les exonérations assèchent les recettes fiscales régionales (villes, villages, hameaux) et amoindrissent les ressources dont disposent les communautés pour soutenir leurs administrés dans le besoin. Par ailleurs, l'absence d'indemnisations financières (une solution pourtant suggérée dans le rapport de décembre 1896 , et qui continuera à faire débat ${ }^{89}$ ) laisse la population dans le dénuement.

Les mesures du gouvernement n'apaisent que brièvement la colère des protestataires avant que les troubles ne reprennent. Le 26 septembre 1898, une nouvelle marche est organisée. Elle sera suivie d'une quatrième le 13 février 1900, qui donnera lieu à de violents affrontements avec la police au lieu-dit de Kawamata 川俣, et se soldera par une centaine d'arrestations et des poursuites judiciaires. Les conséquences des décisions prises par le gouvernement en 1897 alimenteront le conflit pendant encore de nombreuses années.

\section{Conclusion}

Étudier en détail la période 1896-1897 permet de mieux comprendre la position des autorités centrales confrontées à l'affaire d'Ashio. Non interventionnistes durant la première décennie du conflit, puis mises sous pression par une résistance

88. ShōjI \& Sugai 2014 [1984], p. 76-81.

89. Des députés demandent au gouvernement d'arbitrer des négociations entre l'industrie Furukawa et la population en vue de dédommagements, mais sans succès. Ibid., p. 77-78. 
grandissante, elles finissent par s'impliquer graduellement. L'instauration d'une Commission d'enquête interministérielle, et surtout la prise de dispositions, sont les signes tangibles de cette évolution. En suivant au jour le jour les débats entre les commissaires, on peut apprécier les forces en présence et les rapports entre les différentes tendances, mais surtout mettre en lumière les enjeux bien plus larges qui entourent cette crise : l'opposition entre les intérêts de l'agriculture et ceux d'une industrie en pleine expansion, mais sans véritables garde-fous ; la mise en place d'un État centralisé et d'un système juridique moderne faisant ses premiers pas face à des questions de responsabilité civile ; le rôle de l'expertise scientifique dans les décisions politiques. À ce titre, les explications déployées par Gotō, convaincu que la pollution minière n'est pas un danger pour la santé, sont d'autant plus édifiantes que celui-ci assure une fonction centrale dans les politiques de santé publique et la création de l'hygiène industrielle ${ }^{90}$. Face aux intérêts d'un secteur extractif tenu pour vital dans le développement du pays, le sort des habitants des terres contaminées passe au second plan. Gotō joue ainsi pleinement le rôle du technocrate dans ce dossier où pouvoir étatique et expertise scientifique s'imbriquent de façon complexe.

Comme on a pu le constater, l'intervention de l'État dans l'affaire d'Ashio n'a pas eu les mêmes implications pour tous les acteurs. Pour Furukawa, l'exécution des travaux exigés ne comportait finalement pas de grands désavantages. Au prix d'investissements certes massifs, il parvint à protéger son exploitation et ne fut pas obligé d'indemniser les victimes. Ce fut l'État, à travers les exemptions fiscales, qui se chargea d'une partie des dégâts causés aux terres cultivables. L'arrêté du 27 mai 1897 scella ainsi une sorte d'accord tacite entre Furukawa et des autorités acquises à la cause de l'industrialisation - qu'elles encadraient quelque peu, mais ne remettaient certainement pas en question malgré son coût humain et environnemental. Pour la population des zones contaminées, les décisions des autorités centrales eurent en revanche des suites très graves : la pollution persista, les exemptions fiscales privèrent leurs bénéficiaires de droits de vote, et appauvrirent les communautés susceptibles de les soutenir. Des conséquences qui expliquent en partie la poursuite de la lutte au-delà de 1897. L'intervention

90. Sur ces questions, se référer à THOMAnN, 2015. 


\section{CIPANGO}

238 Cahiers d'études japonaises n²3

étatique croissante se traduisit davantage par une répression du mouvement de protestation que par une régulation de l'industrie, notamment à la suite de l'incident de Kawamata. ainsi que par le recours à des moyens radicaux. Pour parer à la colère populaire, le gouvernement instaura en 1902 une nouvelle Commission d'enquête, qui suggéra dès l'année suivante un vaste plan d'aménagement territorial avec la canalisation de la rivière Watarase, du fleuve Tone, et la destruction du village de Yanaka pour faire place à un bassin de sédimentation ${ }^{91}$. Les antagonismes se durcirent davantage à cette occasion, mais le gouvernement ne craignit pas d'être plus coercitif et procéda à des expulsions forcées. Au lieu d'intervenir sur la mine, on canalisa le réseau hydrique, considéré comme la cause première des dégâts, passant ainsi à une échelle d'action supérieure, avec un véritable remodelage du territoire.

En définitive, le gouvernement finit par jouer un rôle prépondérant dans l'affaire d'Ashio, contrairement à des cas analogues de la même période où la négociation s'engagea directement entre les protestataires et l'exploitant, débouchant parfois sur des arrangements plus favorables aux premiers. Le conflit qui débuta dans les années 1890 autour des activités minières de la compagnie Sumitomo, dans le Shikoku, est généralement cité en exemple ${ }^{92}$ : la raffinerie installée près de Niihama, puis déplacée vers la mer Intérieure du Japon, à Shisakajima (département d'Ehime), suscita l'opposition des habitants de la région. Dès 1910, la compagnie accepta finalement d'adapter sa production et de verser des réparations. Indépendamment de leur médiatisation, d'autres conflits environnementaux survinrent dans l'archipel tout au long de la période moderne, avant que le débat ne s'impose à nouveau à partir des décennies 1950-196093. De fait, les protestations continuèrent de manière

91. Cette phase est plus étudiée que la période 1896-1897. Voir notamment MiURA, 2017, p. 184-226.

92. Morris-Suzuki, 1998, p. 764-768 ; Watanabe, 2013.

93. On retient pour cette période quatre grandes maladies qui donnèrent lieu à des actions en justice : la maladie dite de Minamata (empoisonnement au mercure), qui se déclara dans la ville du même nom ainsi que dans le département de Niigata (maladie de Minamata à Niigata), celle appelée itai itai (« aïe aie », infection au cadmium), et l'asthme lié à la pollution atmosphérique de la ville de Yokkaichi. 
sporadique à Ashio, y compris pendant les années 1910-1920 et la Guerre de l'Asie et du Pacifique, tandis que l'opposition à la pollution se manifesta plus largement à travers le pays. Par exemple, la situation japonaise interne et externe n'empêcha pas les pêcheurs de Taiji de porter plainte en 1942 contre l'usine Ajinomoto de l'entreprise Suzuki à Kawasaki ${ }^{94}$. On peut donc considérer que les questions environnementales sont indissociables du développement industriel de l'archipel, et que leur régulation - aboutie ou manquée - contribue à définir les contours de l'État moderne. Quand Carol Gluck revient sur la question de la « modernité » et de ces différentes déclinaisons à travers le monde, l'affaire d'Ashio lui sert à illustrer les « changements cumulatifs en contexte » qui ont fait en sorte qu'un certain type de modernité politique et technologique émerge de l'expérience japonaise ${ }^{95}$. Par l'étude de cet épisode de l'histoire de Meiji, et avec ses différentes implications, nous espérons avoir fourni une pièce supplémentaire pour analyser le rôle de l'État japonais à l'égard des questions industrielles, écologiques et sociales des dernières décennies du XIX ${ }^{\mathrm{e}}$ siècle. Ceci, dans la double finalité d'intégrer ce cas à l'histoire mondiale de la pollution industrielle durant l'époque moderne ${ }^{96}$, et de mettre en regard la réponse du gouvernement japonais avec celle des autres puissances industrialisées.

\section{Bibliographie}

AxaIshi Naomi 赤石直美, 2009, « Tochi daichō ni shirusareta kindai no shizen saigai e no taiō»土地台帳に記された近代の自然災害への対応 [Les réponses aux désastres naturels de l'époque moderne telles qu'enregistrées dans les cadastres], in Kyōto rekishi saigai kenkyu 京都歴史災害研究 [Études

94. Morris-SUZUKI, 1998, p. 768-771.

95. Que ce soit au plan micro (la situation spécifique des campagnes de Tochigi, la modification du système administratif local et de perception des impôts) ou macro (l'industrialisation du pays, le rôle stratégique de la production de cuivre, l'adoption d'une constitution). GLUCK, 2011.

96. Sur le cas français, on se réfèrera entre autres à LE Roux, 2011. 


\section{CIPANGO \\ 240 Cahiers d'études japonaises n²3}

des désastres historiques à Kyōto], $n^{\circ}$ 10, Ritsumeikan daigaku rekishi toshi bōsai kenkyūjo 立命館大学歴史都市防災研究所, Kyōto, pp. 7-12.

ANDō Seiichi 安藤精一, 1992, Kinsei kōgaishi no kenkyū 近世公害史の研 究 [Étude sur l'histoire de la pollution à l'époque pré-moderne], Yoshikawa kōbunkan 吉川弘文館, Tōkyō, 402 p.

AnZai Kunio 安在邦夫, Horiguchi Osamu 堀口修 \& FukUi Atsushi 福井 淳 (ed.), 2009, Ashio dōzan kōdoku jiken kankei shiryō : Kokuritsu kōbun shokan shozo eiinbon 足尾銅山鉱毒事件関係資料: 国立公文書館所蔵影印本 [Matériaux relatifs à l'affaire de pollution de la mine de cuivre d'Ashio : facsimilés des documents détenus par les Archives nationales du Japon], Tōkyō daigaku shuppan-kai 東京大学出版会, Tōkyō, 30 vol.

Enомото Takeaki 榎本武明 \& Kabayama Sukenori 樺山資紀，1938， «Shūgiin giin Tanaka Shōzō-kun hoka yonjūrokumei teishutsu kōeki ni yūgai no kōgyō o teishi sezaru gi ni kan shi shitsumon ni tai suru tōbensho » 衆 議院議員田中正造君外四十六名提出公益に有害の鉱業を停止せざ る義に関し質問に対する答弁書 [Réponse à la question du député à la Chambre des Représentants Tanaka Shōzō et de quarante-six confrères sur la nécessité d'interrompre les activités minières portant préjudice à l'intérêt général], in Nagashima Yohachi 永島与八 (ed.), Ködoku jiken no shinsō to Tanaka Shōzo ō 鉱毒事件の真相と田中正造翁 [Le vieux Tanaka Shōzō et la vérité sur la pollution minière], Meiji bunken 明治文献, Tōkyō, pp. 173-178.

Gluck Carol, 2011, "The End of Elsewhere: Writing Modernity Now" in American Historical Review, n ${ }^{\circ}$ 3, vol. 116, pp. 676-687.

ITSUKA-KaI 五日会 (ed.), 1926, Furukawa Ichibe óden 古河市兵衛翁伝 [Mémoires du vieux Furukawa Ichibē], Itsuka-kai 五日会, Tōkyō, 289 p. 
Kano Masanao 鹿野政直 (ed.), 1974, Ashio kōdoku jiken kenkyū 足尾鉱毒事件 研究 [Études sur l'incident de pollution minière d'Ashio], San.ichi shobō 三 一書房, Tōkyō, 502 p.

Kōdokushi Hensan IInkaI 鉱毒史編纂委員会 [Comité d'édition de l'histoire minière] (ed.), 2006-2013, Kōdokushi 鉱毒史 [Histoire de la pollution minière], Ōta 太田, 1475 p. \& 3015 p.

Komatsu Hiroshi 小松裕, 2001, Tanaka Shōzō no kindai 田中正造の近代 [La modernité de Tanaka Shōzō], Gendai kikakushitsu 現代企画室, Tōkyō, $836 \mathrm{p}$.

Konishi Tokuō 小西徳應, 1989, «Ashio dōzan onzon no kōzō : daisankai kōdoku yobō kōji meirei o chūshin ni » 足尾銅山温存の構造一第 3 回 鉱毒予防工事命令を中心に [Comment on sauvegarda la mine de cuivre d'Ashio : autour de la troisième série de travaux préventifs], in Seikei ronsō 政経論叢 [Collection d'essais en politique et en économie], vol. 58, n 3 et 4, Meiji daigaku seiji keizai kenkyūjo 明治大学政治経済研究所, Tōkyō, pp. 741-798.

Konishi Tokuō 小西徳應, 1990a, « Ashio dōzan kōdoku jiken kenkyū : daisankai kōdoku yobō kōji no jisshi to meireisho no kaizan »足尾銅山 鉱毒事件研究一第 3 回鉱毒予防工事の実施と命令書の改ざん [Recherches sur l'affaire de pollution au cuivre de la mine d'Ashio : la mise en œuvre du troisième plan de travaux préventifs et la falsification de l'arrêté], in Seikei ronsōo 政経論叢 [Collection d'essais en politique et en économie], vol. 58, Meiji daigaku seiji keizai kenkyūjo 明治大学政治経済研究所, Tōkyō, pp. 935-987.

Konishi Tokuō 小西徳應, 1990b, « Ashio kōdoku jiken to seifu : maboroshi no kōgyō-hō to daiichiji kōdoku chōsa iinkai o chūshin ni » 足尾鉱毒事 件と政府一幻の鉱業法と第1次鉱毒調査委員会を中心に [L'affaire d'Ashio et le gouvernement : sur la chimère des lois minières et la première Commission d'enquête sur la pollution minière], in Meiji daigaku shakai 


\section{CIPANGO}

242 Cahiers d'études japonaises n²3

kagaku kenkyūjo kiyo 明治大学社会科学研究所紀要 [Annales de l'Institut des Sciences sociales de l'université Meiji], vol. 28, nº 2, Meiji daigaku seiji keizai kenkyūjo 明治大学政治経済研究所, Tōkyō, pp. 49-77.

LE Roux Thomas, 2011, Laboratoire des pollutions industrielles. Paris, 1770-1830, Albin Michel, Paris, 552 p.

Miura Ken.ichirō 三浦顕一郎, 2017, Tanaka Shōzō to Ashio kōdoku mondai : tsuchi kara umareta riberaru demokurash $\bar{i}$ 田中正造と足尾鉱毒問題: 土か ら生まれたリベラル・デモクラシー [Tanaka Shōzō et le problème de pollution minière d'Ashio : la démocratie libérale née du sol], Yūshisha 有志 舎, Tōkyō, $312 \mathrm{p}$.

Morris-Suzuki Tessa, 1998, "Environmental Problems and Perceptions in Early Industrial Japan”, in Elvin Mark \& Ts'ui-Jung Liu (eds.), Sediments of Time. Environmental Society in Chinese History, Cambridge University Press, New York, pp. 756-780.

Murakami Yasumasa 村上安正, 2006, Ashio dōzan-shi 足尾銅山史 [Histoire de la mine d'Ashio], Zuisōsha 随想舎, Utsunomiya 宇都宮, 654 p.

Nimura Kazuo 二村一夫, 1988, Ashio bōdō no shiteki bunseki : kōzan rōdōsha no shakaishi 足尾暴動の史的分析：鉱山労働者の社会史 [Analyse historique de l'émeute d'Ashio. Histoire sociale des ouvriers de la mine], Tōkyō daigaku shuppankai 東京大学出版会, Tōkyō, 366 p.

Nimura Kazuo 二村一夫, 1997, The Ashio Riot of 1907. A Social History of Mining in Japan, transl. Boardman Terry \& Gordon Andrew, Duke University Press, Durham \& London, 275 p.

Notehelfer Fred G., 1975, “Japan's First Pollution Incident” in Journal of Japanese Studies, n ${ }^{\circ}$, vol. 1, pp. 351-383. 
SHImizu Miyuki 清水みゆき, 1995, Kindai Nihon no han kōgai undō shiron 近 代日本の反公害運動史論 [Essai historique sur les mouvements contre la pollution dans le Japon moderne], Nihon keizai hyōronsha 日本経済評論 社, Tōkyō, 215 p.

Shōjr Kichirō 東海林吉郎 \& Sugar Masurō 菅井益郎, 1984, Tsūshi Ashio kōdoku jiken 通史・足尾鉱毒事件：1877 1984 [Histoire de l'affaire de pollution minière d'Ashio, 1877-1984], Seori shobō 世織書房, Yokohama, $323 \mathrm{p}$.

Sippel Patricia, 2006, “Technology and Change in Japan's Modern Copper Mining Industry”, in Hunter Janet \& STORz Cornelia (eds.), Institutional and Technological Change in Japan's Economy. Past and Present, Routledge, London \& New York, pp. 10-26.

SōGyō Hyakunenshi Hensan IInKaI 創業100年史編纂委員会 [Comité, 1976, Sógyō byakunenshi 創業 100 年史 [Histoire du centenaire de la fondation], Furukawa kōgyō 古河鉱業 [Industrie minière Furukawa], Tōkyō, $768 \mathrm{p}$.

Souyri Pierre-François, 2016, Moderne sans être occidental. Aux origines du Japon d'aujourd'bui, Gallimard, Paris, 490 p.

TAKAGi Kiyoshi 高木潔, 1974, 《Dō to Nihon shihon shugi » 銅と日本資 本主義 [Le cuivre et le capitalisme japonais], in KANo Masanao 鹿野政 直 (dir.), 1974, Ashio ködoku jiken kenkyū 足尾鉱毒事件研究 [Études sur l'incident de pollution minière d'Ashio], San.ichi shobō 三一書房, Tōkyō, pp. 6-41.

TANAKa Shōzō 田中正造, 1989, « Kōeki ni yūgai no kōgyō o teishi sezaru gi ni tsuki shitsumon sho 》公益二有害ノ鉱業习停止セザル儀二付質 問書 [Question sur la nécessité d'interrompre les activités minières portant préjudice à l'intérêt général], in Tanaka Shōzō senshū 田中正造選集 [OEuvres choisies de Tanaka Shōzō], Iwanami shoten 岩波書店, Tōkyō, pp. 53-58. 


\section{CIPANGO}

244 Cahiers d'études japonaises n²3

Thomann Bernard, 2015, La Naissance de l'État social japonais. Biopolitique, travail et citoyenneté dans le Japon impérial (1868-1945), Presses de Sciences Po, Paris, 450 p.

TOCHIGI-KENSHI HENSAN IINKAI 杤木県史編さん委員会 [Comité d'édition de l'histoire du département de Tochigi] (ed.), 1980, Tochigikenshi, shiryō-hen kingendai 杤木県史. 史料編近現代 [Histoire du département de Tochigi. Édition de sources de la période moderne et contemporaine], vol. 9, Ashio 足 尾, $1123 \mathrm{p}$.

Uchimizu Mamoru 内水護 (ed.), 1971, Shiryō Ashio kōdoku jiken 資料足尾 鉱毒事件 [Documents sur l'affaire d'Ashio], Aki shobō 亜紀書房, Tōkyō, $500 \mathrm{p}$.

Watanabe Takehiro, 2013, "Talking Sulfur Dioxide. Air Pollution and the Politics of Science in Late Meiji Japan", in Miller Ian Jared, Thomas Julia Adeney \& Walker Brett L. (eds.), Japan at Nature's Edge. The Environmental Context of a Global Power, University of Hawai'i Press, Honolulu, pp. 73-89.

Yomiuri shinbun, 14 avril 1897, « Gotō eisei-kyokuchō no sankōhin wa mukō nari » 後藤衛生局長の参考品は無効なり [Les échantillons du directeur du bureau de l'Hygiène Gotō ne valent rien], édition de Tōkyō.

Résumé : l'affaire d'Ashio constitua l'une des crises écologiques et sociales majeures du Japon moderne. À partir de 1896, les problèmes environnementaux et la contestation avaient pris une ampleur telle que les autorités centrales furent obligées de s'impliquer, après une décennie de laissez-faire. Le 24 mars 1897 une Commission d'enquête sur la pollution minière d'Ashio fut instituée par le ministère de l'Agriculture et du Commerce, en charge du dossier. Si les mesures prises par le gouvernement représentèrent une étape dans l'encadrement des activités minières, elles avaient surtout pour but la protection de cette industrie. 
L'analyse des procès-verbaux de cette Commission ainsi que des décisions gouvernementales nous renseigne sur la façon dont les autorités de l'époque entendent gérer une crise environnementale, de même que sur les enjeux de l'exploitation d'une ressource naturelle.

Mots-clés : Ashio, Commission d'enquête, pollution, mine de cuivre, environnement

Abstract: The Ashio copper mine case was one of the most important ecological and social crisis of modern Japan. Due to the scale of the environmental issues and of the protest movement, in 1896, the central authorities were forced to get involved, after a decade of laissez-faire. On March 24, 1897, an Ashio pollution investigation Commission was established by the Ministry of Agriculture and Commerce, in charge of this matter. If the measures taken by the Government represented a step towards the supervision of mining activities, the initial purpose was to protect this industry. The analysis of the minutes of the Commission and of the governmental decisions tells us a great deal about how the authorities back then handled an environmental crisis, as well as what was at stake in the exploitation of a natural resource.

Keywords: Ashio, investigation Commission, pollution, coppermine, environment キーワード：足尾，調查委員会，銅山，公害，環境，鉱毒事件 
